\title{
Research on Electromagnetic Interference for Vehicle-mounted Fire Protection System during Engine Ignition
}

\author{
Chunxiao Ren ${ }^{a}$, Guosheng Zhang ${ }^{b}$ \\ Research Institute of Highway Ministry of Transport, Beijing 100088, China \\ a431478306@qq.com, briohrcx@foxmail.com
}

Keywords: EMC; Engine Ignition; Vehicle-mounted Device.

\begin{abstract}
Base on the problem that during the moment of ignition, electromagnetic environment in vehicles could cause interference to the stability and reliability of vehicle fire protection system, causing failure of the fire-prevention function. In this paper, the interference mechanism and harmfulness were analyzed through the simulation experiments. The targeted software design and prevention measures for electromagnetic interference such as shielding, grounding to protect the system were employed, after experiment, the effectiveness of these measures was proved.
\end{abstract}

\section{Introduction}

With the rapid development of automobile industry and electronic technique, more embedded electronic devices are utilized in vehicle, especially in large passenger buses, which participating in complication of vehicle electromagnetic environment. Ensuring electronic devices could meet the EMC requirements during driving is important. The Advanced Driver Assistant Systems technology develops rapidly, the electromagnetic interference problem specially needs to be solved.

Among all kinds of electromagnetic environment, the high frequency radiation caused by vehicle ignition system could interfere other electronic device easily. Vehicle safety system such as fire prevention system and ADAS device became necessarily. In compulsory standard GB18565 of China, the automatic fire extinguisher must be installed in engine compartment. But these kinds of electronic device could be interfered in the complex electromagnetic environment, resulting in function failure. To improve the reliability of these safety device, then ensure the safety of transportation, studies on the interference in ignition moment and propose corresponding solutions are important.

\section{Interference Analysis}

\subsection{Interference Source Analysis.}

Ignition system is an important part of engine which consists of power supply, ignition coil, interrupter and ignition plug. The basic operation principle of the engine starting is that the ignition coil transfers the power supply voltage to high voltage $15-20 \mathrm{kv}$, with the help of distributor, ignition plug generate spark, the flammable gas air is lighted, the engine starts to work.

After analysis of principle of ignition system and feature of vehicle circuit, at the ignition moment the EMI of ignition system are mainly caused:

(1) High frequency EMW generated radiated by ignition coil spreads outward, meanwhile, the voltage near ignition plug is higher than breakdown voltage, resulting in high-pressure discharge and high frequency EMW radiation. This is the main resource of EMI.

(2) During ignition, high current generates in circuit. Usually, the current is more than100A. Transient current in circuit leads to electromagnetic radiation. Since the circuits of vehicle are complicated and various, considerable electromagnetic interference will be caused.

\subsection{Interfered Spot Analysis.}

Fire prevention system consists of signal collection and transform module, CPU and operation module, the working principle is monitoring vehicle environment including the information of 
environmental temperature and smoke, dealing with these signals and reacts accordingly by means of alarming and quenching.

According to the analysis of principle of ignition system and signal transmission form, there are two main spots are interfered usually:

(1) Signal input: the signal collected by the system was transformed into voltage signal and transferred to CUP, during the process, the signal could be interfered, which leads to fluctuation in voltage.

(2) Chip pins: chip pins, especially the suspended pins could be easily interfered by electromagnetic wave, resulting in the anomaly in input and output features, affecting the function of chips and the system.

\section{Experiment Analysis}

The experiment mainly concerned the radiated interference generated at the moment of ignition, simulating continuous interfere environment through the repeatedly ignition of vehicle ignition simulation equipment. The experimental subject, the Vehicle-mounted Fire Protection System was put in ignition simulation equipment, both of them were covered with shielding cover to form separated space. FPS was supplied with $24 \mathrm{~V}$ power, temperature signal collected through the temperature acquisition module as input, the alarm port of operate module was connected with oscilloscope, collecting the signal waveform. The aim of this experiment was to study the interference of vehicle fire prevention system during ignition, through experiment, analyzing the EMC damage from ignition, proposing the solution.

After the experiment, the result showed:

(1) The reference waveform without interference from ignition were acquired firstly. The operation was carried out when the ignition simulation equipment was closed, setting up system, make sure that every module at proper functioning. Making the system work separately in normal monitoring status and alarm status, collecting the output signal waveform. The results were showed in Fig. 1 and Fig. 2.

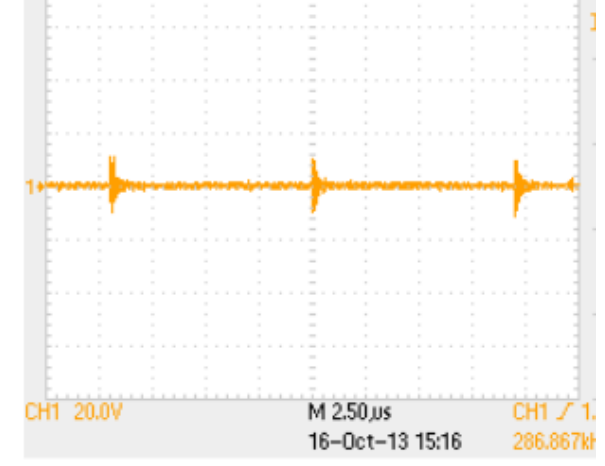

Fig. 1 Output waveform in normal monitoring status

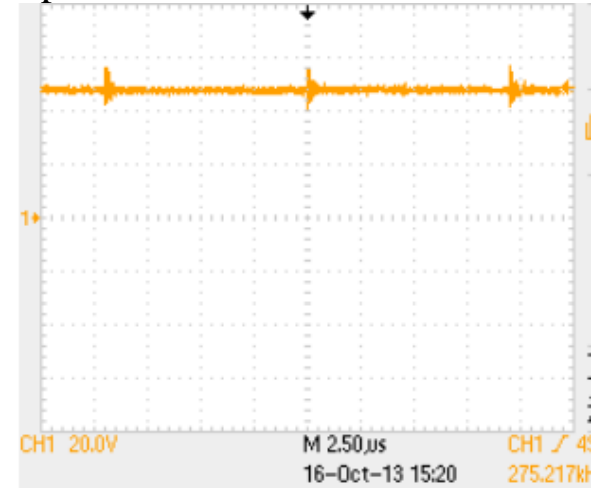

Fig. 2 Output waveform in alarm status

As shown above, when there was no ignition interference, system outputted stable low level signal waveform in normal monitoring state. In alarm state, a high level signal was outputted. 
(2) In order to explore the impact on the fire protection system which came from the ignition interference, the system was putted to work under the condition of opening and closing the ignition simulation equipment respectively. The output waveforms under this two kinds of conditions were collected, which were shown in Fig. 3, Fig. 4.

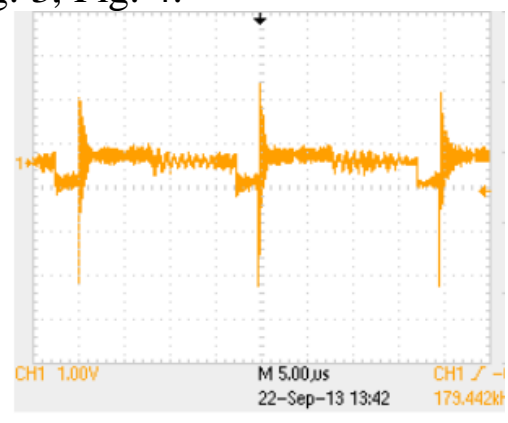

Fig. 3 Output waveform without interference

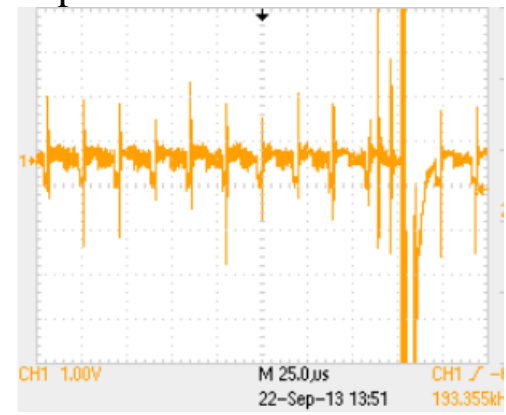

Fig. 4 Output waveform with interference

According to results of the experiment above, the output wave shown in Fig. 4 may be acquired. Comparing Fig. 3 and Fig. 4, showing that the EMI produced at the moment of ignition caused some peak pulse signals. The probable causing of this problem is that high frequency electromagnetic coupling or the input and output characteristics of the chip pins in system host was interfered during signal collection and transmission. This interference will mainly lead to two kinds of consequence.

(1) System false alarm: Comparison of experiment 1 and 2 shows that, even though system don't meet the condition of alarm, it will also produce high-amplitude pulse because of the effect of EMI, which can lead to system's wrong operation. The actual consequence is the causing that airbag pop-up or alarm for no reason.

(2) System damage: EMF constantly interferes chip electrical pins, long-term effect may decrease sensitivity of chip pins, and cause input-output characteristic abnormality, then make the system malfunction, that in the event of abnormal conditions which require some appropriate measures, system give no response.

\section{Solutions}

From the above experimental results, during vehicle ignition, EMI will directly impact the stability and reliability of vehicle safety system, also, could cause damage to the system, so, measures must be taken to protect the system. For hardware, there are mainly two widely used technologies in addition to some anti-jamming design while PCB making, which are shielding and grounding technology.

\subsection{Hardware Design}

Electromagnetic shielding could cut off the way of electromagnetic coupling. Shielding could prevent EMW moving from one space to another by absorbing and reflecting energy. However, not every kind of shielding boxes are fully applicable to ignition interference of vehicles. The system shielding box were designed using the following two methods.

(1) Material selection: Metal has good effects for absorbing and loss of electromagnetic energy. If the conductivity and permeability of the metal are stronger, and the frequency of the EMW is higher, the power of metal absorbing electromagnetic wave is stronger. As high frequency radiation 
interference, the ignition interference, whose frequency of is in the range of $10 \mathrm{kHz}-1000 \mathrm{MHz}$, needs aluminum shielding box for protection.

(2) Gaps and holes: The shielding effectiveness of the shielding box not only depends on the material, but also depends on the design of slits and holes of the box. Shielding box adopts the structure of five surfaces integrated into one whole, minimizing the gaps. Making the side which is perforated and seamed away from the engine compartment. Reducing leakage by minimizing the screw holes. Covering the wire connected to the fire protection system host with the shielded line, and use shielding material seal the connection between system and wire.

There are two main methods of grounding: single-point grounding and multi-point grounding. As the normal vehicle safety systems are low frequency circuits with the frequency less than $1 \mathrm{MHz}$, the inductance impact between wiring and devices is not critical, but the loop current impact on the interference needs more concern. So, single-point grounding method was proposed, which means all devices use a common signal ground. This has been designed when PCB making.

Combining grounding and shielding technology, the shielding box was connected to the car body, the "quiet place" was accomplished. Shielding capacity of shielding box was strengthened not only by reflecting and absorbing EMW, but also by leading the excess magnetic energy into "quiet place".

\subsection{Software Design}

Software design for anti-interference is on the premise that system itself is not affected. Recognizing and processing the interference input signal by programming, false alarm could be avoided, the anti-interference ability of the system improved effectively.

Delay trigger: As the ignition interference occurs only during vehicle ignition, so interference signals often appear as peak pulse signals. Therefore, the method of delay processing could be adopted, it means using twice judgments to determine whether the signal was the fire signal, as the flowchart of software following.

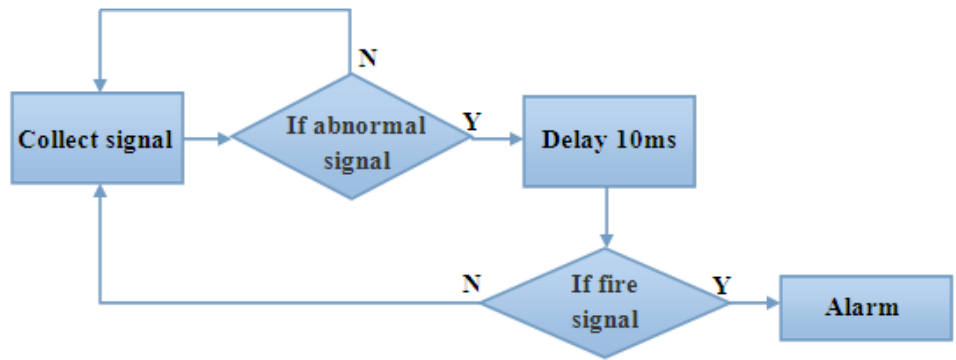

Fig. 5 Flowchart of delay trigger

This method is simple and effective. The disadvantage is that delay will increase the response time of system in case of fire.

By combine using the method of weakening and eliminating, dealing those peak pulse signals. Weakening method made the high-amplitude abnormal signal weakened. Eliminating method replaced the interference signals. Setting the sample frequency $100 \mathrm{~Hz}$, sampling 100 times, the average of 100 times samples was taken as an effective signal for transmission, named $S_{a}$. Every sample signal was determined whether it was abnormal by comparing the difference value between current and previous signal, if the difference value was bigger than alarm temperature gradient threshold $\mathrm{M}$, it could be judged abnormal. If the abnormal signal captured, named $S_{n}$, calculating the difference value between $S_{n}$ and the next signal $S_{n+1}$, the method to determine it was interfered is that whether the difference value was bigger than the alarm temperature gradient threshold $\mathrm{M}$ of the system. If the signal confirmed interference, eliminate it. Give the average of $S_{n-1}$ and $S_{n+1}$ to Sn. Flowchart of determination follows. 


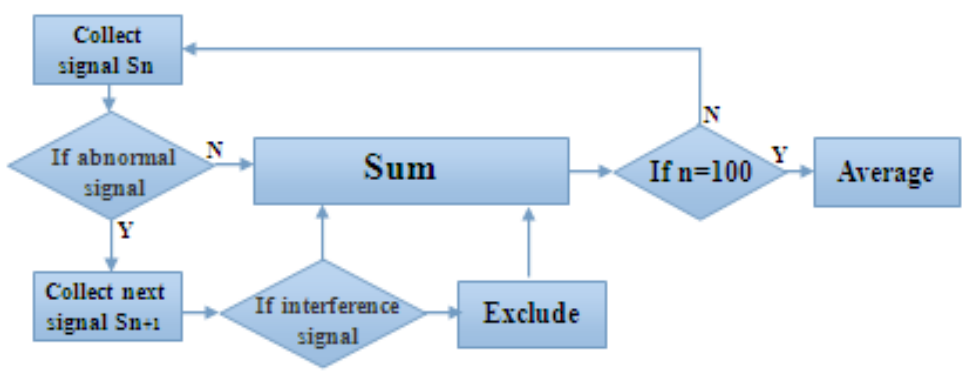

Fig. 6 Flowchart of excluding and weakening

The above filtering and processing for the original collected signals of system, not only improved the reliability of the collected signals for system, but also eliminated the effect which comes from interference signals to the final decision of the system more thoroughly.

\section{Effect Verification}

After the application of grounding and shielding and the software design, the result of verification experiment shows that the stability of the signal of the fire prevention system has been improved. The result of the experiment was shown in Fig. 7.

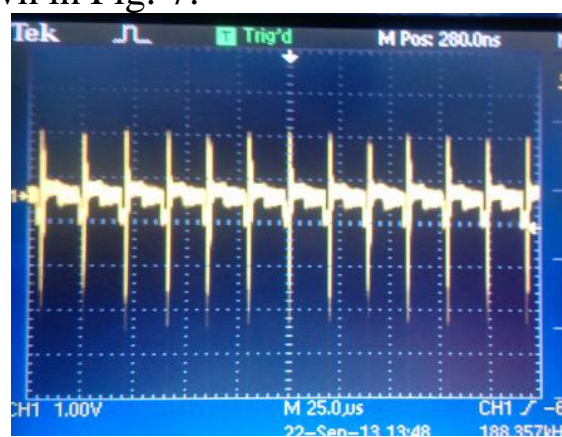

Fig. 7 Output signal after interference protection

\section{Summary}

Focusing on the problem that the electromagnetic environment could cause interference to the vehicle fire protection systems when vehicle ignition, this paper studied the source of EMI and the serious consequences by experiment analysis. Through the analysis of the types and caused of EMI, Software and hardware measures were respectively proposed to protect and improve those onboard systems. The effectiveness of those measures was proved.

\section{References}

[1]. Lan Bo, Sun Yi. The Development Status of Automobile EMC Research. Information Technology. Vol. 8(2010), p. 210-212.

[2]. Zheng Yali, Yu Jihui, Wang Quandi, et al. Dynamic Circuit Model of the Spark Plug for EMC Prediction of Ignition System. Transactions of China Electrotechnical Society. Vol. 26(2011), p. 8-10.

[3]. Wang Quandi, Liu Chunyan, Yu Jihui, et al. 2007. On Suppressing Electromagnetic Interference Caused by Automobile Spark-ignition System. Journal of Chongqing University (Natural Science Edition). Vol. 30(2007), p. 46-49.

[4]. Li Yanchun, Sheng Yihua. Research on Grounding and Disturbance Restraint in Electromagnetic Compatibility. Shanxi Electric Power. Vol. 3(2009), p. 57-59. 